\title{
Efficacy of Adalimumab in Korean Patients with Crohn's Disease
}

\author{
II Woong Sohn, Sung Tae Kim, Bun Kim, Hyun Jung Lee, Soo Jung Park, Sung Pil Hong, Tae II Kim, Won Ho Kim, and Jae \\ Hee Cheon \\ Division of Gastroenterology and Institute of Gastroenterology, Department of Internal Medicine, Yonsei University College of Medicine, Seoul, \\ Korea
}

Background/Aims: Adalimumab is effective for both remission induction and the maintenance of Crohn's disease (CD) in Western countries. We evaluated the efficacy of adalimumab in the conventional step-up treatment approach for CD in Korea. Methods: We retrospectively reviewed 62 patients with CD who were treated with adalimumab. Their Crohn's disease activity index (CDAI) was measured at weeks 4, 8, and 52. Clinical remission was defined as a CDAl score $<150$. Induction and maintenance outcomes were analyzed. Results: Forty-one patients (66.1\%) achieved a reduction of 70 CDAl points at week 8. Among them, 28 (45.2\%) achieved clinical remission at week 8, 20 (32.3\%) maintained remission at week 52 . The absence of prior anti-tumor necrosis factor (TNF) therapy and Montreal classification L1 at baseline predicted clinical remission at week 8 in the multivariate logistic regression analysis. In the Cox proportional hazards model, the hazard ratio for the secondary loss of response during maintenance therapy after clinical remission induction was significantly higher in patients who showed initial mild CDAl severity or Montreal classification A3. Conclusions: In our study, anti-TNF therapy-naive and Montreal classification L1 were associated with adalimumab efficacy as induction therapy in CD. Further studies are warranted to determine the prognostic factors for the long-term response after adalimumab therapy. (Gut Liver 2016;10:255-261)

Key Words: Crohn disease; Adalimumab; Remission induction; Treatment outcome

\section{INTRODUCTION}

Crohn's disease (CD) is a chronic idiopathic inflammatory bowel disorder and its disease course fluctuates between clini- cal remission and relapse. A considerable number of patients eventually require bowel resection and postoperative recurrence is very common. ${ }^{1}$ The conventional approach for induction and maintenance treatment for $\mathrm{CD}$ has changed since new biologic agents have been introduced. Monoclonal anti-tumor necrosis factor (TNF) antibodies have become a mainstay for CD treatment during the last few decades.

In Asia, anti-TNF agents are used less frequently than they are in Western countries because of their limited, strict indications under insurance coverage rules and the social economic burden. ${ }^{2}$ Of the anti-TNF agents, infliximab and adalimumab were approved in Korea in 2003 and 2010, respectively. Infliximab, a chimeric monoclonal immunoglobulin G1 antibody against TNF- $\alpha$, was the first biologic agent approved for the treatment of CD in Korea. However, 10\% to 30\% of patients are primary nonresponders to infliximab, and its annual drop-out rate has been reported to be $20 \%$ to $40 \%$ because of the loss of response or occurrence of side effects. ${ }^{3}$ One of the important causes of loss of response is the development of anti-infliximab antibodies. ${ }^{4}$ Adalimumab has become a new treatment option for patients with $\mathrm{CD}$ who are either naive to, failed to respond to, or experienced loss of response to infliximab therapy. ${ }^{5-9}$ Adalimumab, a recombinant, fully humanized immunoglobulin G1 monoclonal antibody, is theoretically less vulnerable to immunogenicity development. This drug has proved effective for remission induction and maintenance in Western CD patients. Despite the therapeutic efficacy of adalimumab, its efficacy in Korean patients is still unknown, and suitable patient characteristics and the appropriate timing for administration with regard to phenotypic behavior, disease distribution, and disease activity have yet to be determined. Therefore, in the present study, we aimed to evaluate the efficacy of adalimumab in Korean patients with $\mathrm{CD}$ in a single tertiary academic center and deter-

Correspondence to: Jae Hee Cheon

Division of Gastroenterology and Institute of Gastroenterology, Department of Internal Medicine, Yonsei University College of Medicine, 50-1 Yonsei-ro, Seodaemun-gu, Seoul 120-752, Korea

Tel: +82-2-2228-1990, Fax: +82-2-393-6884, E-mail: geniushee@yuhs.ac

Received on April 6, 2015. Revised on May 24, 2015. Accepted on June 12, 2015. Published online October 19,2015 pISSN 1976-2283 eISSN 2005-1212 http://dx.doi.org/10.5009/gnl15165

@ This is an Open Access article distributed under the terms of the Creative Commons Attribution Non-Commercial License (http://creativecommons.org/licenses/by-nc/4.0) which permits unrestricted non-commercial use, distribution, and reproduction in any medium, provided the original work is properly cited. 
mine the predictors of a favorable response.

\section{MATERIALS AND METHODS}

\section{Patients}

A total of 62 patients with CD who were treated with adalimumab at a tertiary academic center in Korea between October 2008 and December 2014 were included in this retrospective study. CD was diagnosed based on the assessment of location and extent of chronic intestinal mucosal inflammation through endoscopy with biopsies and radiological testing. The allowable indications for adalimumab therapy based on the National Health Insurance of Korea are as follows: (1) patients who become nonresponsive to conventional therapy, including corticosteroids and immunomodulators; (2) patients with moderate to severe CD (Crohn's disease activity index [CDAI] $\geq 220$ ) who were contraindicated for conventional therapy; and (3) patients who have failed infliximab treatment.

Nonsmokers were defined as those who currently do not smoke cigarettes including both former smokers (ex-smokers) and never smokers. The Montreal classification was used for assessing disease location and behavior. ${ }^{10}$ Patients with evidence of tuberculosis exposure via a protein-purified derivative skin test or interferon $\gamma$ releasing assay for tuberculous antigen and a chest X-ray received antituberculosis prophylactic treatment before the initiation of adalimumab therapy. Adalimumab was contraindicated for patients with serious concurrent infection or malignancy, such as lymphoproliferative disorders.

Our study had ethical approval from the Severance Institutional Review Board, but the requirement for informed consent was waived due to its retrospective design.

\section{Assessment of disease activity}

Most controlled clinical trials for $\mathrm{CD}$ define clinical remission as CDAI <150 points (CR-150) and clinical response as a reduction in CDAI of 70 points (CR-70) or a reduction in CDAI of 100 points (CR-100) from baseline. ${ }^{11}$ CDAI score was assessed at weeks 4,8 , and 52 . The proportion of patients achieving clinical remission $(\mathrm{CDAI}<150)$ as well as the proportion of patients achieving CR-70 and CR-100 was assessed.

Data from all patients who achieved clinical remission $(\mathrm{CDAI}<150)$ at the end of the 8 -week induction trial were analyzed with a univariate analysis. Patients who showed a clinical response, regardless of degree, at the end of the 8-week induction trial were enrolled in the analysis of the maintenance trial. Total follow-up time was determined from the start of therapy to the last date of adalimumab administration or adalimumab discontinuation.

\section{Primary and secondary outcome analyses}

The primary outcome analysis was to determine the proportion of patients with an initial treatment response to and treat-
Table 1. Patient Demographic and Baseline Characteristics

\begin{tabular}{|c|c|}
\hline Characteristic & Value \\
\hline \multicolumn{2}{|l|}{ Sex } \\
\hline Male & $36(58.1)$ \\
\hline Female & $26(41.9)$ \\
\hline Age at anti-TNF induction, yr & $30.4 \pm 9.5(15-52)$ \\
\hline \multicolumn{2}{|l|}{ Duration of $\mathrm{CD}$, yr } \\
\hline$\leq 2$ & $6(9.7)$ \\
\hline $2-5$ & $12(19.4)$ \\
\hline$>5$ & $44(71.0)$ \\
\hline Weight, kg & $51.0 \pm 10.6(34.0-87.0)$ \\
\hline Tobacco usage, nonsmoker & $58(93.5)$ \\
\hline \multicolumn{2}{|l|}{ Extraintestinal manifestation } \\
\hline Arthritis & $3(4.8)$ \\
\hline Erythema nodosum & $3(4.8)$ \\
\hline Aphthous stomatitis & $4(6.5)$ \\
\hline Iritis/uveitis & $4(6.5)$ \\
\hline \multicolumn{2}{|c|}{ Montreal classification, age at diagnosis } \\
\hline $\mathrm{A} 1$ & $12(19.4)$ \\
\hline A2 & 49 (79.0) \\
\hline A3 & $1(1.6)$ \\
\hline \multicolumn{2}{|l|}{ Montreal classification, location } \\
\hline L1 & $14(22.6)$ \\
\hline $\mathrm{L} 2$ & $4(6.5)$ \\
\hline L3 & 44 (71.0) \\
\hline L4 & $5(8.1)$ \\
\hline
\end{tabular}

Montreal classification, behavior

B1

$39(62.9)$

B2

$10(16.1)$

B3

$13(21.0)$

Perianal disease

27 (43.5)

CDAI

BMI, $\mathrm{kg} / \mathrm{m}^{2}$

$301.0 \pm 79.3$ (151.0-568.7)

CRP, $\mathrm{mg} / \mathrm{L}$

$18.4 \pm 3.3(13.6-29.3)$

$21.5 \pm 24.3(0.2-115.8)$

$\mathrm{ESR}, \mathrm{mm} / \mathrm{hr}$

$51.0 \pm 31.9(2.0-120.0)$

WBC, $/ \mathrm{mm}^{3}$

Hematocrit, \%

$6,687 \pm 2,483(2,510-15,510)$

$36.7 \pm 5.5(21.8-47.5)$

Albumin, g/dL

$3.5 \pm 0.6(2.0-4.6)$

Indication of adalimumab use

Both anti-TNF agent and

0

azathioprine-naive

Anti-TNF agent-naive+azathioprine

$21(33.9)$

Inflixmab failure

$41(66.1)$

Data are presented as mean \pm SD (range) or number (\%). TNF, tumor necrosis factor; CD, Crohn's disease; CDAI, Crohn's disease activity index; BMI, body mass index; CRP, C-reactive protein; ESR, erythrocyte sedimentation rate; WBC, white blood cell. 
ment maintenance with adalimumab. The secondary outcome analysis was to assess the predictors associated with inducing and maintaining clinical remission.

\section{Statistical analysis}

All statistical analyses were performed using SPSS version 18.0 (SPSS Inc., Chicago, IL, USA), and a two-sided p-value $<0.05$ was considered to be statistically significant. The chisquare test or Fisher exact test was used for the comparison of categorical data to determine clinical factors predictive of clinical response at week 8 , and odds ratios (ORs) were provided where necessary.

The sustained clinical benefit of adalimumab was estimated with Kaplan-Meier analyses. To compare hazard ratios defined by one variable at the time, the log-rank test was used. Logistic and Cox proportional hazard models were conducted to detect predictors of clinical response to adalimumab treatment.

Table 2. Univariate Analysis of Variables for Clinical Remission at Week 8

\begin{tabular}{|c|c|c|c|c|c|}
\hline Variable & Remission $(\mathrm{n}=28)$ & Nonremission $(\mathrm{n}=34)$ & OR & 95\% CI & $\mathrm{p}$-value \\
\hline Male sex & $21(75.0)$ & $15(44.1)$ & 3.800 & $1.277-11.312$ & 0.014 \\
\hline Nonsmoker & $24(85.7)$ & $34(100.0)$ & 2.471 & $1.779-3.283$ & 0.023 \\
\hline \multicolumn{6}{|l|}{ Disease duration, yr } \\
\hline$\leq 2$ & $4(14.3)$ & $5(14.7)$ & 2.667 & $0.451-15.781$ & 0.265 \\
\hline $2-5$ & $7(25.0)$ & $27(79.4)$ & 1.933 & $0.539-6.937$ & 0.307 \\
\hline$>5$ & $17(60.7)$ & $12(35.3)$ & 0.401 & $0.130-1.235$ & 0.107 \\
\hline \multicolumn{6}{|l|}{ Induction regimen, $\mathrm{mg}$} \\
\hline $160 / 80$ & $15(53.6)$ & $12(35.3)$ & 2.115 & $0.761-5.883$ & 0.149 \\
\hline $80 / 40$ & $13(46.4)$ & $22(64.7)$ & 0.453 & $0.163-1.257$ & 0.126 \\
\hline History of bowel resection & $11(39.3)$ & $20(58.8)$ & 0.453 & $0.163-1.257$ & 0.126 \\
\hline Naive to anti-TNF agents & 15 (53.6) & $6(17.6)$ & 5.385 & $1.700-17.054$ & 0.003 \\
\hline \multicolumn{6}{|l|}{ Montreal classification } \\
\hline \multicolumn{6}{|l|}{ Age a diagnosis } \\
\hline A1 & $8(28.6)$ & $4(11.8)$ & 3.000 & $0.796-11.308$ & 0.096 \\
\hline A2 & $20(71.4)$ & $29(85.3)$ & 0.431 & $0.123-1.511$ & 0.182 \\
\hline A3 & 0 & $1(2.9)$ & 0.541 & $0.429-0.682$ & 0.360 \\
\hline \multicolumn{6}{|l|}{ Disease location } \\
\hline L1 & $10(35.7)$ & $4(11.8)$ & 4.167 & $1.137-15.265$ & 0.025 \\
\hline L2 & $2(7.1)$ & $2(5.9)$ & 1.231 & $0.162-9.344$ & 0.841 \\
\hline L3 & $16(57.1)$ & $28(82.4)$ & 0.286 & $0.090-0.908$ & 0.030 \\
\hline L4 & $3(10.7)$ & $2(5.9)$ & 1.920 & $0.298-12.384$ & 0.487 \\
\hline \multicolumn{6}{|l|}{ Disease behavior } \\
\hline B1 & $17(60.7)$ & $22(64.7)$ & 0.843 & $0.300-2.372$ & 0.746 \\
\hline B2 & $7(25.0)$ & $3(8.8)$ & 3.444 & $0.799-14.855$ & 0.085 \\
\hline B3 & $4(14.3)$ & $9(26.5)$ & 0.463 & $0.126-1.706$ & 0.241 \\
\hline Perianal disease & $10(35.7)$ & $17(50.0)$ & 0.556 & $0.199-1.548$ & 0.259 \\
\hline \multicolumn{6}{|l|}{ Medication } \\
\hline Azathioprine & $16(57.1)$ & $12(35.3)$ & 2.333 & $0.832-6.543$ & 0.105 \\
\hline Prednisolone & $5(17.9)$ & $3(8.8)$ & 2.303 & $0.802-6.610$ & 0.312 \\
\hline 5-ASA & $23(82.1)$ & 30 (88.2) & 0.460 & $0.100-2.126$ & 0.312 \\
\hline \multicolumn{6}{|l|}{ Disease activity } \\
\hline Mild & $4(14.3)$ & $2(5.9)$ & 2.667 & $0.451-15.781$ & 0.265 \\
\hline Moderate & 24 (85.7) & 30 (88.2) & 0.800 & $0.181-3.536$ & 0.768 \\
\hline Severe & 0 & $2(5.9)$ & 0.533 & $0.421-0.676$ & 0.192 \\
\hline
\end{tabular}

Data are presented as number (\%).

OR, odds ratio; CI, confidence interval; TNF, tumor necrosis factor; 5-ASA, 5-aminosalicylate. 


\section{RESULTS}

\section{Patient baseline characteristics}

Baseline clinical characteristics of 62 patients with CD (36 male; mean age, 30.4 years; range, 15 to 52 years) are shown in Table 1. Their mean baseline CDAI score was 301; 9.7\% of patients had mild disease activity $(150 \leq \mathrm{CDAI}<220), 87.1 \%$ had moderate disease activity $(220 \leq \mathrm{CDAI}<450)$, and $3.2 \%$ had severe disease activity (CDAI $\geq 450)$ at enrollment. The six patients who were administered adalimumab despite low disease activity had undergone multiple bowel resections. They were at high risk for developing short bowel syndrome and other intestinal complications. Eight patients received antituberculosis prophylactic treatment before the initiation of adalimumab therapy. Fortyone of 62 patients $(66.1 \%)$ had previously failed to respond to or had experienced loss of response to infliximab.

Six patients (9.7\%) presented with at least one extra-intestinal manifestation at baseline. Three patients (4.8\%) had peripheral arthralgia, four (6.5\%) had ocular manifestations, four (6.5\%) had aphthous stomatitis, and the remaining three (4.8\%) had erythema nodosum.

\section{Outcomes at week 8}

Of the 62 patients included in this analysis, three (4.8\%) discontinued adalimumab therapy by week 8 due to primary nonresponse. Twenty-eight patients (45.2\%) showed complete remission, 35 (56.5\%) showed CR-100, and 41 (66.1\%) showed CR-70 by week 8 . The remaining 18 patients (29.0\%) continued receiving adalimumab therapy in an effort to achieve a delayed response, although their initial responses were considered unsatisfactory.

To determine factors predictive of clinical remission at week 8, univariate unadjusted ORs were calculated (Table 2). Male sex, nonsmoker, naive status to an anti-TNF agent, and ileal disease were associated with clinical remission at week 8. Twenty-eight patients (45.2\%) received combination therapy with adalimumab and azathioprine, which did not increase the complete remission rate at week 8 on univariate analysis $(p=0.105)$.

A multivariate analysis revealed that naive status to an antiTNF agent (OR, 5.120; 95 confidence interval [CI], 1.416 to 18.509) and ileal disease (OR, 4.708; 95\% CI, 1.087 to 20.391) were independent predictors of clinical remission at week 8 (Table 3).

Table 3. Multivariate Logistic Regression Analysis of Predictors Associated with Clinical Remission at Week 8

\begin{tabular}{lccc}
\hline \multicolumn{1}{c}{ Predictor } & OR & $95 \%$ CI & p-value \\
\hline Naive to biological agent & 5.120 & $1.416-18.509$ & 0.013 \\
Montreal classification, L1 & 4.708 & $1.087-20.391$ & 0.038 \\
Montreal classification, A1 & 4.202 & $0.914-19.321$ & 0.065 \\
Induction regimen, 160/80 mg & 1.834 & $0.529-6.366$ & 0.339 \\
\hline
\end{tabular}

OR, odds ratio; CI, confidence interval.

\section{Outcomes at week 52}

In total, 38 patients $(61.3 \%)$ were still being treated with adalimumab by the end of follow-up. Twenty patients (32.3\%) showed complete remission, 28 (45.2\%) showed CR-100, and 32 (51.6\%) showed CR-70 by 52 weeks (Fig. 1 ).

On univariate analysis, Montreal classification A3 and mild disease severity were associated with loss of response at week 52 ( $\mathrm{p}=0.019$ and $\mathrm{p}=0.013$, respectively) (Table 4). On multivariate analysis, Montreal classification A3 and mild disease activity were independently associated with clinical relapse at week 52 (Table 5).

Thirty-six patients (58\%) received concurrent azathioprine therapy, which did not affect treatment outcome (log-rank test, $\mathrm{p}=0.484$ ). Two patients developed herpes zoster during adalimumab therapy, but life-threatening side effects did not occur during the adalimumab treatment.

\section{DISCUSSION}

The purpose of this study was to evaluate the efficacy of adalimumab as induction and maintenance therapy for CD in Korea. Therapeutic strategies in CD patients treated with adalimumab have not been well evaluated compared to those with infliximab. Under the step-up approach, patients who are unresponsive and intolerant to 5-aminosalicylate, corticosteroids, azathioprine, and/or infliximab are switched to adalimumab therapy. Adalimumab became an additional treatment option in patients who had lost response to or were intolerant to conventional treatments. In addition, adalimumab can be used before infliximab therapy in patients with failure to respond to conventional therapy.

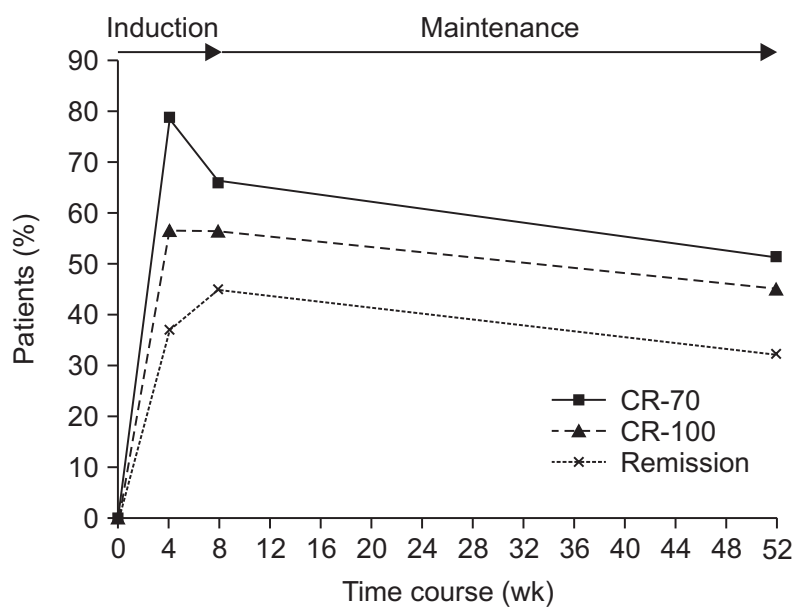

Fig. 1. Clinical response to and remission rates after adalimumab therapy.

CR-70, clinical response based on a reduction of 70 points in the Crohn's disease activity index (CDAI); CR-100, clinical response based on a reduction of 100 points in the CDAI from baseline; Remission, CDAI $<150$ points. 
Table 4. Univariate Analysis of Variables for Clinical Relapse during Adalimumab Therapy (Log-Rank Test)

\begin{tabular}{|c|c|}
\hline Predictor & p-value \\
\hline Male sex & 0.074 \\
\hline \multicolumn{2}{|l|}{ Duration of Crohn's disease, yr } \\
\hline$\leq 2$ & 0.185 \\
\hline $2-5$ & 0.288 \\
\hline$>5$ & 0.701 \\
\hline Tobacco use, nonsmoker & 0.645 \\
\hline \multicolumn{2}{|l|}{ Montreal classification, age at diagnosis } \\
\hline A1 & 0.508 \\
\hline $\mathrm{A} 2$ & 0.970 \\
\hline A3 & 0.019 \\
\hline \multicolumn{2}{|l|}{ Montreal classification, location } \\
\hline L1 & 0.422 \\
\hline L2 & 0.886 \\
\hline L3 & 0.568 \\
\hline L4 & 0.698 \\
\hline \multicolumn{2}{|l|}{ Montreal classification, behavior } \\
\hline B1 & 0.856 \\
\hline B2 & 0.281 \\
\hline B3 & 0.550 \\
\hline Perianal disease & 0.096 \\
\hline History of bowel resection & 0.893 \\
\hline \multicolumn{2}{|l|}{ Medication at administration of $\mathrm{ADA}$} \\
\hline Azathioprine & 0.105 \\
\hline Prednisolone & 0.547 \\
\hline 5-ASA & 1.000 \\
\hline \multicolumn{2}{|l|}{ CDAI severity } \\
\hline Mild & 0.013 \\
\hline Moderate & 0.064 \\
\hline Severe & 0.726 \\
\hline Prior use of infliximab & 0.955 \\
\hline \multicolumn{2}{|l|}{ Induction regimen, mg } \\
\hline $160 / 80$ & 0.279 \\
\hline $80 / 40$ & 0.279 \\
\hline \multicolumn{2}{|l|}{ Indications for therapy } \\
\hline Drug resistant & 0.226 \\
\hline Penetrating disease & 0.367 \\
\hline Deep ulcer and extensive disease & 0.353 \\
\hline
\end{tabular}

ADA, adalimumab; 5-ASA, 5-aminosalicylate; CDAI, Crohn's disease activity index.

In our study, 35 of 62 patients (56.5\%) achieved clinical response (change in the $\mathrm{CDAI} \geq 100$ ), including 28 (45.2\%) who achieved complete remission at week 8 of the induction therapy. More than half of patients with CD showed an initial clinical benefit from adalimumab therapy as an induction therapy.

The induction benefits of adalimumab for patients with active
Table 5. Cox Predictive Factors for Clinical Relapse during Adalimumab Therapy

\begin{tabular}{lclc}
\hline \multicolumn{1}{c}{ Predictor } & HR & \multicolumn{1}{c}{$95 \%$ CI } & p-value \\
\hline Montreal A3 & 17.231 & $1.670-177.838$ & 0.017 \\
Mild CDAI severity & 7.520 & $1.666-33.948$ & 0.009 \\
\hline
\end{tabular}

HR, hazard ratio; CI, confidence interval; CDAI, Crohn's disease activity index.

CD were first demonstrated in the CLASSIC (Clinical Assessment of Adalimumab Safety and Efficacy Studied as an Induction Therapy in Crohn's) I trial. The remission rate at week 4 for patients administered the higher 160/80 mg adalimumab dosing was significantly different from that for patients receiving placebo ( $36 \%$ vs $12 \%$, respectively, $\mathrm{p}=0.001$ ). Clinical response rate (change in the $\mathrm{CDAI} \geq 100$ ) for the higher adalimumab dosing schedule was also significantly different from that of placebo at week 4 (50\% for the 160/80 mg adalimumab group vs 25\% for placebo, $\mathrm{p}=0.002) .^{5}$ In this study, the predictors of response to anti-TNF- $\alpha$ therapy in CD included a short duration of disease and inflammatory disease, colonic disease, less severe disease, and nonsmoking. ${ }^{12}$

In our study, nonsmoker, naive status to an anti-TNF agent, and a Montreal classification of L1 for disease location were associated with an increased complete remission rate on univariate analysis. A multivariate analysis revealed that naive status to an anti-TNF agent (OR, 5.120; 95 CI, 1.416 to 18.509) and a Montreal classification of L1 for disease location (OR, 4.708; 95\% CI, 1.087 to 20.391) were independent predictors for clinical remission at week 8. Our results are consistent with those of previous studies in that nonsmokers and patients naive to an anti-TNF agent showed better response during induction therapy. It is well known that patients with a primary nonresponse or loss of response to one anti-TNF agent have a suboptimal response to a second agent. ${ }^{13}$ Patients naive to anti-TNF therapy showed a better response to adalimumab. Clinical remission rates were approximately 53.6\% $(\mathrm{p}=0.003)$ in patients naive to anti-TNF therapy compared with $17.6 \%$ in patients who had been treated with infliximab. These results could be due to a very similar mode of action for both drugs. However, immunogenicity is completely different between the two drugs. Approximately $10 \%$ to $30 \%$ of patients per year discontinue infliximab therapy because of loss of response or side effects. ${ }^{3}$ Introduction of adalimumab after failure of infliximab therapy resulted in sustained clinical benefits in 50\% to $60 \%$ of patients. ${ }^{14}$

It is well known that determining disease behavior and the location of the lesions is of paramount importance at the timing of drug administration. Small bowel and anoperineal involvement at diagnosis were predictors of early structuring or penetrating complications. However, L1 disease was associated with a higher clinical remission rate in our study, which was different from data from other trials. ${ }^{13,15,16}$ The reasons for this discrep- 
ancy are currently unknown and should be further investigated.

The mean duration of follow-up was 19.1 months (range, 1 to 56 months), and the median was 14 months in our study. Maintenance therapy demonstrated continuous clinical remission over 52 weeks in 20 patients (32.3\%). Clinical response (change in the $\mathrm{CDAI} \geq 100$ ) was achieved in 28 patients (45.2\%).

The long duration of the efficacy of adalimumab maintenance therapy in $\mathrm{CD}$ was evaluated in a follow-up randomized controlled trial (CLASSIC II). ${ }^{7}$ Of 204 patients in the preceding CLASSIC I trial, 93 (46\%) were in clinical remission at week 56. Recent post hoc subgroup analyses of the Crohn's Trial of the Fully Human Antibody Adalimumab for Remission Maintenance and Additional Long-term Dosing with Adalimumab to Evaluate Sustained Remission and Efficacy in Crohn's Disease were performed. ${ }^{17}$ Predictors for loss of response or dose escalation were male sex, current/former smoker status, family history of inflammatory bowel disease, isolated colonic disease, extraintestinal manifestations, 80/40 mg induction therapy, longer disease duration, greater baseline CDAI, concomitant corticosteroid use, no deep remission at week 12, low serum trough concentrations of adalimumab, previous infliximab nonresponse, and being previously treated with an anti-TNF agent. ${ }^{18}$

Based on the Kaplan-Meier analysis and log-rank test results in our study, Montreal classification A3 and lower CDAI score were associated with poor long-term outcomes. In the Cox proportional hazards model, the hazard ratio for maintenance of response was significantly higher for patients who showed mild CDAI severity and Montreal classification A3. Further studies are warranted to validate the current findings. One possible explanation is that mild CDAI severity might be related to reasons other than bowel inflammation itself and Montreal classification A3 might reflect more extensive disease. Previous infliximab treatment did not alter clinical course on maintaining clinical remission contrary to inducing clinical remission.

The advantages of adalimumab and azathioprine combination therapy are still debating. Induction and maintenance therapy with azathioprine and adalimumab were shown to have no long-term advantages over adalimumab alone in a recent study. ${ }^{19}$ In our study, both baseline immunomodulator and concurrent immunomodulator combination with adalimumab did not increase the efficacy of anti-TNF therapy (log-rank test, $\mathrm{p}=0.484$ ), which needs further validation.

No patient discontinued adalimumab due to adverse effects. The risk of opportunistic infections increases in patients receiving immunosuppressive medications, including corticosteroids, anti-TNF- $\alpha$ agents, and 6 -mercaptopurine/azathioprine. ${ }^{20}$ Two patients contracted herpes zoster infection, and both of them were taking adalimumab with concurrent immunomodulators. There were no allergic reactions or serious infections. In a metaanalysis of 21 studies including 5,356 individuals, anti-TNF therapy did not increase the risks of death, malignancy, or serious infection. ${ }^{21}$ There was no serious adverse effect in this study.
This is the first study from a Korean population, which is one of the strengths of our study. Moreover, it was investigated in real clinical practice setting. However, there are some limitations of our study, in that it was a retrospective study from a single center. The follow-up might not have been sufficiently long for evaluation. In addition, the assessment of mucosal healing plays a useful role in guiding therapeutic adjustment. Many studies showed a relatively poor correlation with CDAI in Crohn's patients. ${ }^{22}$ The six patients with mild disease activity at induction did not reflect the real disease activity. Our study did not investigate the impact of endoscopy on clinical decision and disease monitoring, but regular endoscopic assessment is impractical in the clinical settings. Finally, only a small number of the overall studied patients were enrolled in our analysis. Multicenter studies with a larger number of patients would be warranted to draw a concrete conclusion to examine the efficacy of adalimumab in Korean patients with $\mathrm{CD}$.

In conclusion, adalimumab is an effective treatment for the induction and maintenance of remission for CD. CD patient naive to anti-TNF therapy and Montreal classification L1 were associated with higher rates of clinical remission. Further studies are warranted to determine the prognostic factors for long-term response.

\section{CONFLICTS OF INTEREST}

No potential conflict of interest relevant to this article was reported.

\section{ACKNOWLEDGEMENTS}

This work was supported by Basic Science Research Program through the National Research Foundation of Korea funded by the Ministry of Science, ICT \& Future Planning (NRF2013R1A2A2A01067123) and grants of the Korean Health Technology R\&D Project, Ministry of Health and Welfare, Republic of Korea (A120176 and HI13C1345).

\section{REFERENCES}

1. Lee YW, Lee KM, Chung WC, Paik CN, Sung HJ, Oh YS. Clinical and endoscopic recurrence after surgical resection in patients with Crohn's disease. Intest Res 2014;12:117-123.

2. Park SJ, Kim WH, Cheon JH. Clinical characteristics and treatment of inflammatory bowel disease: a comparison of Eastern and Western perspectives. World J Gastroenterol 2014;20:1152511537.

3. Schnitzler F, Fidder H, Ferrante M, et al. Long-term outcome of treatment with infliximab in 614 patients with Crohn's disease: results from a single-centre cohort. Gut 2009;58:492-500.

4. Nanda KS, Cheifetz AS, Moss AC. Impact of antibodies to infliximab on clinical outcomes and serum infliximab levels in patients 
with inflammatory bowel disease (IBD): a meta-analysis. Am J Gastroenterol 2013;108:40-47.

5. Hanauer SB, Sandborn WJ, Rutgeerts P, et al. Human anti-tumor necrosis factor monoclonal antibody (adalimumab) in Crohn's disease: the CLASSIC-I trial. Gastroenterology 2006;130:323-333.

6. Colombel JF, Sandborn WJ, Rutgeerts P, et al. Adalimumab for maintenance of clinical response and remission in patients with Crohn's disease: the CHARM trial. Gastroenterology 2007;132:5265.

7. Sandborn WJ, Hanauer SB, Rutgeerts $\mathrm{P}$, et al. Adalimumab for maintenance treatment of Crohn's disease: results of the CLASSIC II trial. Gut 2007;56:1232-1239.

8. Sandborn WJ, Rutgeerts P, Enns R, et al. Adalimumab induction therapy for Crohn disease previously treated with infliximab: a randomized trial. Ann Intern Med 2007;146:829-838.

9. Chang CW, Wei SC, Chou JW, et al. Safety and efficacy of adalimumab for patients with moderate to severe Crohn's disease: The Taiwan Society of Inflammatory Bowel Disease (TSIBD) Study. Intest Res 2014;12:287-292.

10. Silverberg MS, Satsangi J, Ahmad T, et al. Toward an integrated clinical, molecular and serological classification of inflammatory bowel disease: report of a Working Party of the 2005 Montreal World Congress of Gastroenterology. Can J Gastroenterol 2005;19 Suppl A:5A-36A.

11. Van Assche G, Dignass A, Panes J, et al. The second European evidence-based consensus on the diagnosis and management of Crohn's disease: definitions and diagnosis. J Crohns Colitis 2010;4:7-27.

12. Yanai H, Hanauer SB. Assessing response and loss of response to biological therapies in IBD. Am J Gastroenterol 2011;106:685-698.

13. Singh S, Garg SK, Pardi DS, Wang Z, Murad MH, Loftus EV Jr. Comparative efficacy of biologic therapy in biologic-naive pa- tients with Crohn disease: a systematic review and network metaanalysis. Mayo Clin Proc 2014;89:1621-1635.

14. Karmiris K, Paintaud G, Noman M, et al. Influence of trough serum levels and immunogenicity on long-term outcome of adalimumab therapy in Crohn's disease. Gastroenterology 2009;137:1628-1640.

15. Kim ES, Kim WH. Inflammatory bowel disease in Korea: epidemiological, genomic, clinical, and therapeutic characteristics. Gut Liver 2010;4:1-14.

16. Yang DH, Yang SK, Park SH, et al. Usefulness of C-reactive protein as a disease activity marker in Crohn's disease according to the location of disease. Gut Liver 2015;9:80-86.

17. Panaccione R, Colombel JF, Sandborn WJ, et al. Adalimumab maintains remission of Crohn's disease after up to 4 years of treatment: data from CHARM and ADHERE. Aliment Pharmacol Ther 2013;38:1236-1247.

18. Billioud V, Sandborn WJ, Peyrin-Biroulet L. Loss of response and need for adalimumab dose intensification in Crohn's disease: a systematic review. Am J Gastroenterol 2011;106:674-684.

19. Ferrante M, Karmiris K, Newnham E, et al. Physician perspectives on unresolved issues in the use of conventional therapy in Crohn's disease: results from an international survey and discussion programme. J Crohns Colitis 2012;6:116-131.

20. Toruner M, Loftus EV Jr, Harmsen WS, et al. Risk factors for opportunistic infections in patients with inflammatory bowel disease. Gastroenterology 2008;134:929-936.

21. Peyrin-Biroulet L, Deltenre P, de Suray N, et al. Efficacy and safety of tumor necrosis factor antagonists in Crohn's disease: metaanalysis of placebo-controlled trials. Clin Gastroenterol Hepatol 2008;6:644-653.

22. Jones J, Loftus EV Jr, Panaccione R, et al. Relationships between disease activity and serum and fecal biomarkers in patients with Crohn's disease. Clin Gastroenterol Hepatol 2008;6:1218-1224. 